\title{
Selection of high heterozygosity popcorn varieties in Brazil based on SSR markers
}

\author{
I.B.O. Eloi ${ }^{1}$, C.A. Mangolin ${ }^{1}$, C.A. Scapim ${ }^{2}$, C.S. Gonçalves ${ }^{1}$ and \\ M.F.P.S. Machado ${ }^{1}$ \\ ${ }^{1}$ Departamento de Biologia Celular e Genética, \\ Universidade Estadual de Maringá, Maringá, PR, Brasil \\ ${ }^{2}$ Departamento de Agronomia, Universidade Estadual de Maringá, \\ Maringá, PR, Brasil \\ Corresponding author: M.F.P.S. Machado \\ E-mail: mfpsmachado@uem.br
}

Genet. Mol. Res. 11 (3): 1851-1860 (2012)

Received July 13, 2011

Accepted January 13, 2012

Published July 19, 2012

DOI http://dx.doi.org/10.4238/2012.July.19.5

\begin{abstract}
We analyzed genetic structure and diversity among eight populations of popcorn, using SSR loci as genetic markers. Our objectives were to select SSR loci that could be used to estimate genetic diversity within popcorn populations, and to analyze the genetic structure of promising populations with high levels of heterozygosity that could be used in breeding programs. Fifty-seven alleles ( 3.7 alleles per locus) were detected; the highest effective number of alleles (4.21) and the highest gene diversity ( 0.763$)$ were found for the Umc2226 locus. A very high level of population differentiation was found $\left(F_{\mathrm{sT}}=0.3664\right)$, with $F_{\mathrm{ST}}$ for each locus ranging from 0.1029 (Umc1664) to 0.6010 (Umc2350). This analysis allowed us to identify SSR loci with high levels of heterozygosity and heterozygous varieties, which could be selected for production of inbred lines and for developing new cultivars.
\end{abstract}

Key words: Zea mays; Popcorn; SSR locus; Microsatellite; Genetic diversity; Molecular breeding 


\section{INTRODUCTION}

Studies on the genetic variability in popcorn varieties are important due to the great interest of popcorn production in Brazil. There is a lack of information on genetic structure and diversity of the populations in adapted varieties with desirable agronomic traits. In Brazil, popcorn improvement has been considered an important investment to reduce the import of seeds and also for the development of new varieties adapted to different and heterogeneous regions of country (Scapim et al., 2006). There are few genotypes available to supply domestic demand (Scapim et al., 2006; Freitas et al., 2006; Pereira et al., 2008; Rangel et al., 2008; Vilela et al., 2008). Given the continental size of Brazil with different climatic conditions, the release of popcorn hybrids by public and private institutions for each region is very important to reduce dependence on seeds from foreign markets, particularly due to the cultivation of North American hybrids (Miranda et al., 2008; Santos et al., 2008; Vieira et al., 2009; Leonello et al., 2009).

The use of single sequence repeat (SSR) markers in the identification of genetic variability of popcorn germplasm has been reported in lines (Liu et al., 2003; Dandolini et al., 2008; Leal et al., 2010) and in different genotypes (Wu et al., 2004; Silva et al., 2009). The goal of these studies has been mainly to predict heterozygous hybrid combinations in the breeding programs, the number of allele per SSR locus, and the most polymorphic SSR locus. The genetic dissimilarity between lines and genotypes has been reported, but the genetic structure of those populations has not been described. The knowledge of the observed and expected heterozygosity levels and of the differentiation among the popcorn populations would help identify genotypes that have higher genetic diversity within populations for line extraction to improve breeding programs. The lines obtained may be finally used for the generation of new hybrid cultivars. The hybrids from breeding program have a high chance of producing superior lines and unknown popcorn genotypes may also present high potential for line extraction.

Zelia, a North American hybrid, and IAC-112, a modified simple hybrid, are cultivar hybrids from breeding programs, which have been used in line extraction (Miranda et al., 2008; Vieira et al., 2009). The open-pollinated varieties and unknown popcorn genotypes may also be used in line extraction, but it is important that a preliminary evaluation of the base population be conducted to predict their potential for line extraction, use at an experimental level and/or in combination for future release as superior cultivars. Due to the lack of information on genetic structure of populations in cultivar hybrids with desirable agronomic traits and in open-pollinated varieties and unknown popcorn genotypes, in the present study we analyzed the genetic structure and diversity among populations of popcorn using SSR primers as genetic markers and a set of eight genotypes that represent the diversity available currently and in use by breeding programs. The objectives of the present study were: a) selection of SSR loci most suitable for estimating genetic diversity; b) estimation of the genetic diversity within popcorn varieties, and c) to analyze the genetic structure of the promising populations for use in breeding programs.

\section{MATERIAL AND METHODS}

A set of eight popcorn genotypes was chosen to represent part of the germplasm 
available in our collection (UEM: State University of Maringá, PR, Brazil). Selections were made from Zélia (DuPont of Brazil S.A. - Pioneer Seeds) and IAC-112 (Agronomy Institute of Campinas, SP, Brazil) hybrids, from the open-pollinated varieties Viçosa M-21 (Federal University of Viçosa, MG, Brazil), UNB-02 (National University of Brasília, DF, Brazil), SE-013 (hybrid of North American origin), and Laranjeiras do Sul (originated from Taiwan, Republic of China), and from the unknown popcorn genotypes Argentina and Chile. The seeds of 20 plants per genotype were germinated in an acclimatized chamber and DNA was isolated from young leaves of 10 seedlings per genotype according to the Hoisington et al. (1994) procedure.

The 100 SSR primers previously mapped for maize were obtained from http:// www.maizegdb.org and used in 8 or 9 DNA samples (a total of 69 samples) of each popcorn variety. After screening all the primers, a set of 15 polymorphic SSR primers (Table 1) was selected for further analysis.

Table 1. Single sequence repeat primers used for analysis of genetic diversity and structure of populations of the eight varieties of popcorn.

\begin{tabular}{|c|c|c|c|}
\hline Primer & Repeat type & Sequence $\left(5^{\prime} \rightarrow 3^{\prime}\right)$ & Chromosome \\
\hline BNLG1063 & $(\mathrm{AG})_{42}$ & $\begin{array}{l}\text { Forward: GGAGACAACCCCGACGAC } \\
\text { Reverse: GGTACCAGAGCCACAGATCC }\end{array}$ & 2 \\
\hline BNLG1083 & $(\mathrm{AG})_{29}$ & $\begin{array}{l}\text { Forward: ACAGTCTGTTGGGGAACAGG } \\
\text { Reverse: CAACGCTGGTTTGTCGTTTA }\end{array}$ & 1 \\
\hline UMC1071 & $(\mathrm{TACGA})_{5}$ & $\begin{array}{l}\text { Forward: AGGAAGACACGAGAGACACCGTAG } \\
\text { Reverse: GTGGTTGTCGAGTTCGTCGTATT }\end{array}$ & 1 \\
\hline UMC1077 & $(\mathrm{CA})_{15}(\mathrm{CGCA})_{12}$ & $\begin{array}{l}\text { Forward: CAGCCACAGTGAGGCACATC } \\
\text { Reverse: CAGAGACTCTCCATTATCCCTCCA }\end{array}$ & 10 \\
\hline UMC1653 & $(\mathrm{GAAA})_{24}$ & $\begin{array}{l}\text { Forward: GAGACATGGCAGACTCACTGACA } \\
\text { Reverse: GCCGCCCACGTACATCTATC }\end{array}$ & 6 \\
\hline UMC1664 & $(\mathrm{AACA})_{6}$ & $\begin{array}{l}\text { Forward: AATTGTTTACTGCGCTGAAACTCC } \\
\text { Reverse: CCTCTTTGCCTGTACCGTGTATTC }\end{array}$ & 1 \\
\hline UMC1736 & $(\mathrm{GCAT})_{6}$ & $\begin{array}{l}\text { Forward: CCATCCACCACTAGAAAGAGAGGA } \\
\text { Reverse: TTAATCGATCGAGAGGTGCTTTTC }\end{array}$ & 2 \\
\hline UMC2047 & $(\mathrm{GACT})_{4}$ & $\begin{array}{l}\text { Forward: GACAGACATTCCTCGCTACCTGAT } \\
\text { Reverse: CTGCTAGCTACCAAACATTCCGAT }\end{array}$ & 1 \\
\hline UMC2115 & $(\mathrm{TGCCA})_{5}$ & $\begin{array}{l}\text { Forward: CTGTCTGTCTACCCAACCCAACAG } \\
\text { Reverse: GGGGATAGGCGTGTGTATGTACTG }\end{array}$ & 5 \\
\hline UMC2116 & $(\mathrm{GCCG})_{4}$ & $\begin{array}{l}\text { Forward: CGGTCGATATAATCTTGGCTGATT } \\
\text { Reverse: GGCGCAGAGATCATTTGTTTAAT }\end{array}$ & 1 \\
\hline UMC2226 & $(\mathrm{TGG})_{6}$ & $\begin{array}{l}\text { Forward: TGCTGTGCAGTTCTTGCTTCTTAC } \\
\text { Reverse: AGCTTCACGCTCTTCTAGACCAAA }\end{array}$ & 1 \\
\hline UMC2262 & $(\mathrm{CATCT})_{5}$ & $\begin{array}{l}\text { Forward: TCTGTTCGGGATTCTTCTTCAGTC } \\
\text { Reverse: CGTTCCCTGGTACCCTGTCTATAA }\end{array}$ & 3 \\
\hline UMC2281 & $(\mathrm{GTCC})_{5}$ & $\begin{array}{l}\text { Forward: CAATGATTGGAGCCTAACCCCT } \\
\text { Reverse: ATGATGATCTGCAGAGCCTAGTCC }\end{array}$ & 4 \\
\hline UMC2343 & $\left(\right.$ TGTGTGTG) ${ }_{4}$ & $\begin{array}{l}\text { Forward: TCATCTTCCCCACAAATTTTCATT } \\
\text { Reverse: GACTGACAACTCAGATTTCACCCA }\end{array}$ & 9 \\
\hline UMC2350 & $(\mathrm{GGCCGT})_{4}$ & $\begin{array}{l}\text { Forward: AGTAGCGACTCCTCTGCGTGAG } \\
\text { Reverse: CGAATCGAGGATGGTTTGTTTTT }\end{array}$ & 10 \\
\hline
\end{tabular}

Sixty-four primers amplified well for all popcorn samples but 38 were monomorphic for all 69 plants. The polymerase chain reaction (PCR) was performed using a TC512 thermal cycle (TECHNE, Cambridge, UK). The amplifications were performed by the Touchdown PCR program (Don et al., 1991) using volumes of $20 \mathrm{~mL}$ containing $25 \mathrm{ng}$ genomic DNA, $10 \mathrm{mM}$ Tris- $\mathrm{HCl}, \mathrm{pH} 8.8[2.0 \mu \mathrm{L}$ 10X reaction buffer $(20 \mathrm{mM}$ Tris- $\mathrm{HCl}$, pH 8.0, $0.1 \mathrm{mM}$ EDTA, $1.0 \mathrm{mM}$ DTT, 50\% (v/v) glycerol) or $1 \mathrm{X}$ reaction buffer], $2.5 \mathrm{mM}$ 
$\mathrm{MgCl}_{2}, 0.1 \mathrm{mM}$ of each dATP, dGTP, dCTP, dTTP, $0.3 \mathrm{~mL}$ of each primer ( $\mathrm{F}$ and R primers) and $1 \mathrm{U}$ Taq DNA polymerase (Invitrogen, Carlsbad, CA, USA). After amplification, a total of $20 \mu \mathrm{L}$ of the mix (69 samples) were separated by gel electrophoresis on $4 \%$ agarose gel. The agarose gel was prepared with 50\% agarose Metaphor (Cambrex, Rockland, ME, USA) and 50\% standard agarose using $0.5 \mathrm{X}$ TBE buffer (44.5 mM Tris, $44.5 \mathrm{mM}$ boric acid and 1 mM EDTA; Sambrook et al., 1989). All 69 samples amplified by SSR primer were run on the same gel at $60 \mathrm{~V}$ for $4 \mathrm{~h}$. A 1-kb ladder (Invitrogen) was used as a molecular weight marker. Gels were stained using $0.5 \mu \mathrm{g} / \mathrm{mL}$ ethidium bromide and the image was captured by Ultraviolet Transilluminator High Performance-Edas 290 using the 1-D 3.5 program (Kodak, Rochester, NY, USA). The allele numbers per locus were determined based on their relative position on the gel.

Genetic variability in eight varieties of popcorn was analyzed with the POPGENE 1.32 Computer Program (Yeh et al., 1999). The allele frequencies, percentage of polymorphic loci $(\% \mathrm{P}), \chi^{2}$ test for deviation from Hardy-Weinberg equilibrium, observed and expected mean heterozygosities $\left(H_{\mathrm{O}}\right.$ and $\left.H_{\mathrm{E}}\right)$, mean number of alleles per locus $\left(N_{\mathrm{A}}\right)$, mean number of alleles per polymorphic locus $\left(N_{\mathrm{A} / \mathrm{p})}\right)$, and number of effective alleles $\left(N_{\mathrm{E}}\right)$ were estimated for the 15 SSR loci and 8 popcorn varieties. The fixation index $\left(F_{\text {Is }}\right)$ and Wright's (1965) Fstatistic values $\left(F_{\text {IT }}\right.$ and $\left.F_{\text {ST }}\right)$ were also calculated for the 15 SSR loci. Genetic identity (Nei, 1973) and distances among the varieties were calculated by UPGMA grouping.

\section{RESULTS}

Thirty-eight primers were monomorphic for all samples tested and only 26 amplified two or more products (polymorphic loci). The estimated proportion of SSR polymorphic loci $(\% \mathrm{P})$ in the eight varieties is $41 \%$. A total of 57 alleles, or an average of 3.7 alleles per locus, were detected in leaves of the popcorn plants obtained from seeds of the eight varieties (Figure 1; Table 2).

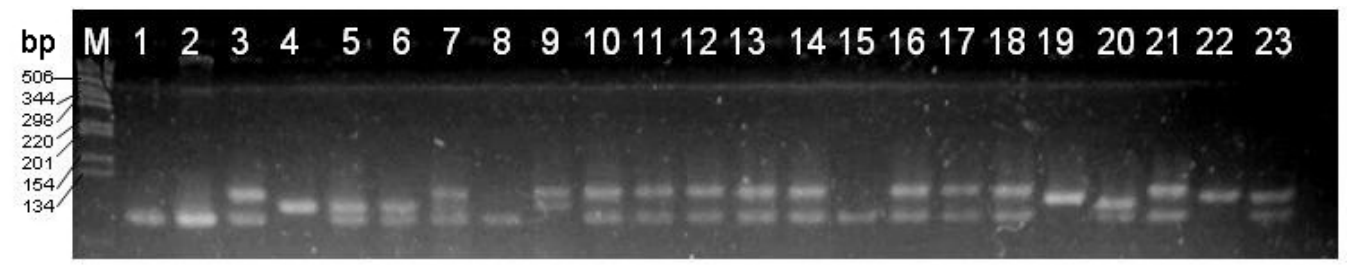

Figure 1. Single sequence repeat fingerprint of the Viçosa (lanes 1-9), IAC-112 (lanes 10-18) and SE-013 (lanes 19-23) popcorn varieties using the UMC1071 primer. Lane $M=$ Molecular weight marker (1-kb ladder of Invitrogen).

An extra allele was evident as a third allele in one plant of Viçosa M-21 and SE-013 varieties using the UMC1653 primer. The extra band (allele 4) was observed as having a comparable intensity with the two original alleles and allele 4 was detected exclusively in the plants from the Laranjeiras do Sul variety, showing an allele frequency equal to 0.1875 . The triallelic phenotype was not considered in the analysis of polymorphism in the SSR loci of popcorn, but it is important to report an extra third allele at the SSR locus of popcorn. A triallelic pattern at microsatellite loci has been observed in other Zea mays cultivars (in su-1 progenies of sweet 


\begin{tabular}{|c|c|c|c|c|c|c|c|}
\hline Locus & $N_{\mathrm{A}}$ & $N_{\mathrm{E}}$ & $H_{\mathrm{O}}$ & $H_{\mathrm{E}}$ & $F_{\text {Is }}$ & $F_{\mathrm{IT}}$ & $F_{\text {ST }}$ \\
\hline Bnlg1063 & 4.0 & 2.3505 & 0.1594 & 0.5746 & 0.4230 & 0.7159 & 0.5077 \\
\hline Bnlg1083 & 5.0 & 2.5981 & 0.2319 & 0.6151 & 0.4004 & 0.6306 & 0.3840 \\
\hline Umc1071 & 4.0 & 2.1231 & 0.4928 & 0.5290 & -0.2240 & 0.0635 & 0.2349 \\
\hline Umc1077 & 4.0 & 3.4689 & 0.3188 & 0.7117 & 0.2313 & 0.5466 & 0.4102 \\
\hline Umc1653 & 5.0 & 1.4936 & 0.2464 & 0.3305 & 0.0035 & 0.2611 & 0.2585 \\
\hline Umc1664 & 3.0 & 2.2274 & 0.8261 & 0.5510 & -0.6778 & -0.5051 & 0.1029 \\
\hline Umc1736 & 3.0 & 1.9094 & 0.2174 & 0.4763 & 0.1159 & 0.5225 & 0.4599 \\
\hline Umc2047 & 3.0 & 2.4855 & 0.2754 & 0.5977 & 0.1936 & 0.5438 & 0.4343 \\
\hline Umc2115 & 3.0 & 2.4155 & 0.5217 & 0.5860 & -0.0312 & 0.1067 & 0.1338 \\
\hline Umc2116 & 3.0 & 2.3669 & 0.4928 & 0.5775 & -0.2115 & 0.1324 & 0.2839 \\
\hline Umc2226 & 5.0 & 4.2189 & 0.6232 & 0.7630 & -0.2695 & 0.1749 & 0.3500 \\
\hline Umc2262 & 4.0 & 3.1985 & 0.4348 & 0.6874 & -0.0643 & 0.3560 & 0.3949 \\
\hline Umc2281 & 4.0 & 3.1540 & 0.7246 & 0.6829 & -0.5196 & -0.0592 & 0.3030 \\
\hline$U m c 2343$ & 4.0 & 3.5306 & 0.2029 & 0.7168 & 0.3100 & 0.7125 & 0.5833 \\
\hline Umc2350 & 2.0 & 1.8304 & 0.1159 & 0.4537 & 0.3518 & 0.7414 & 0.6010 \\
\hline Mean & 3.7 & 2.6248 & 0.3923 & 0.5902 & -0.0563 & 0.3307 & 0.3664 \\
\hline
\end{tabular}

corn; 8\%, e.g.) using the UMC1446, UMC1590 and UMC2343 primers (Rupp et al., 2009). The triallelic pattern in SSR loci has been considered evidence of chimeras, a mechanism of genetic diversification well characterized by Hocquigny et al. (2004) occurring in grapevine clones (Crespan, 2004; Moncada et al., 2006). The detection of 3 alleles at the same locus could be the result of a chimeric structure in which genotypes of different cell layers displayed different alleles. However, as the chimeric situation was not clearly investigated in our study, the third allele at Umc1653 locus was not considered to estimate the polymorphism.

The $N_{\mathrm{E}}$ is not equivalent among the $15 \mathrm{SSR}$ loci. The highest $N_{\mathrm{E}}(4.21)$ and the highest $H_{\mathrm{E}}(0.763)$ were detected in the Umc2226 locus with repeated motifs of three nucleotides [(TGG) $)_{6}$, and a lower $N_{\mathrm{E}}(1.49)$ and lower $H_{\mathrm{E}}(0.33)$ was detected in the Umc1653 locus with repeated motifs of four nucleotides [(GAAA) $)_{24}$ (Table 2). The mean $H_{\mathrm{E}}$ of all SSR loci is 0.5902 .

The larger $N_{\mathrm{E}}$ per locus and the higher $H_{\mathrm{E}}(>0.50)$ detected in 12 SSR loci (Umc1071, Umc2262, Umc2281, Bnlg1063, Bnlg1083, Umc1077, Umc2226, Umc2343, Umc2115, Umc2116, Umc2047, and Umc1664) suggest that $80 \%$ of the 15 SSR loci are adequate and sufficient to make differentiation of the 8 popcorn varieties. Allele frequencies were analyzed for the 15 SSR loci. Departure from Hardy-Weinberg equilibrium was observed in 37 of the 92 (40.2\%) tests done in the eight varieties (data not shown) owing to an excess of heterozygous individuals. The $F_{\text {Is }}$ value was positive in $53.3 \%$ of the SSR loci. However, an excess of heterozygous plants was detected $(5.63 \%)$ for all SSR loci in the eight varieties. The $F_{\text {Is }}$ value was negative at Umc1071, Umc2262,Umc2281, Umc2226, Umc2115, Umc2116, and Umc1664 loci, indicating an excess of heterozygous plants in $46.66 \%$ of loci. Excess of heterozygous plants occurred mainly in the Zelia, IAC-12, Argentina, and UNB-02 varieties (Table 3).

An extremely high population differentiation level was detected in samples from all eight varieties of popcorn $\left(F_{\mathrm{sT}}=0.3664\right)$ with $F_{\mathrm{sT}}$ for each locus ranging from 0.1029 (Umc1664) to $0.6010(U m c 2350)$. The $F_{\text {ST }}$ value indicated that $36.64 \%$ of the total variance in allele frequencies at SSR loci was due to genetic differences among the eight varieties of popcorn.

Table 3 shows the proportion of $N_{\mathrm{A} / \mathrm{pl}}$, mean $H_{\mathrm{O}}$ and $H_{\mathrm{E}}$ in the eight varieties of popcorn. The highest $H_{\mathrm{O}}$ value $(0.5567)$ was found in the UNB-02 variety whereas the lowest value (0.2074) was recorded in the Chile variety. The highest $H_{\mathrm{E}}$ value was 0.5547 for the Laran- 


\begin{tabular}{|c|c|c|c|c|c|c|c|}
\hline Genotype & $\mathrm{N}$ & $N_{\mathrm{A} / \mathrm{pl}}$ & $N_{\mathrm{A}}$ & $N_{\mathrm{E}}$ & $\% \mathrm{P}$ & $H_{\mathrm{O}}$ & $H_{\mathrm{E}}$ \\
\hline Zélia & 8 & 9 & 1.7333 & 1.5471 & 60.00 & 0.4417 & 0.2599 \\
\hline IAC-112 & 9 & 11 & 1.8667 & 1.5477 & 73.33 & 0.4148 & 0.2790 \\
\hline Viçosa M-21 & 9 & 15 & 2.9333 & 2.0065 & 100.0 & 0.3852 & 0.4539 \\
\hline UNB-02 & 8 & 15 & 3.0000 & 2.4322 & 100.0 & 0.5667 & 0.5521 \\
\hline SE-013 & 9 & 13 & 2.8667 & 2.1301 & 86.67 & 0.3852 & 0.4650 \\
\hline Laranjeiras do Sul & 8 & 15 & 3.1333 & 2.3607 & 100.00 & 0.4833 & 0.5547 \\
\hline Argentina & 9 & 7 & 1.4667 & 1.3746 & 46.67 & 0.2889 & 0.2053 \\
\hline Chile & 9 & 7 & 1.7333 & 1.5002 & 46.67 & 0.2074 & 0.2346 \\
\hline
\end{tabular}

jeiras do Sul variety and the lowest $H_{\mathrm{E}}$ value $(0.2053)$ was recorded in the Argentina variety. The Argentina and Chile varieties had the lowest values for mean $H_{\mathrm{O}}(0.2889$ and 0.2074 , respectively), since eight of the 15 SSR loci are in homozygosis for Argentina (Umc1063, Umc1653, Umc1071, Umc2262, Umc2350, Umc1077, Umc2343, Umc2047) and Chile (Umc1653, Umc1071, Umc2350, Umc1736, Umc2226, Umc2343, Umc2047, Umc1664); different alleles were detected only at seven SSR loci (46.67\%). A tendency for allele fixation was evident for Argentina and Chile varieties, which have unknown genealogy. Despite being hybrids, the Zélia and IAC-112 varieties also showed a tendency for allele fixation at six and four SSR loci, respectively.

\section{DISCUSSION}

In this study, we have shown that the estimated proportion of polymorphic loci in the eight varieties of popcorn was $41 \%$ and that $64 \%$ of the primers derived from field maize (26 showing polymorphisms) selected from genomic libraries and databases can be efficiently applied to amplification and analysis of genetic structure and diversity of popcorn. A high number of primers derived from field maize have yet to be used to characterize the genetic diversity of Brazilian popcorn (Dandolini et al., 2008; Silva et al., 2009; Trindade et al., 2010). Our results enable us to stimulate the use of primers for SSR loci derived from field maize for the analysis of genetic structure and diversity among popcorn populations. Null alleles were not detected for the eight varieties of popcorn from the analyses of the 15 primers (13 UMC and 2 BNLG).

The mean number of alleles estimated in the eight varieties of popcorn ( 3.7 alleles/ locus) was higher than the values reported for lines from popcorn and field maize. Li et al. (2004) reported 2.7 alleles per locus in 56 lines of popcorn using 113 primers and 3.35 and 3.0 alleles/locus were reported by Dandolini et al. (2008) and Trindade et al. (2010), respectively, using 15 primers in 10 and 15 lines of popcorn. For 21 lines of field maize, Li et al. (2004) reported 3.58 alleles per locus using 113 SSR primers. As expected, open-pollinated populations outcrossing a higher allele mean-number (5.4) have been detected in popcorn varieties using 61 primers (Wu et al., 2004). In the open-pollinated field maize cultivars, the analysis of 50 SSR loci by Liu et al. (2005) and 45 SSR loci by Qi-Lun et al. (2008) showed a mean number of alleles equal to 4.1 and 6.4, respectively. The evidence in the present study indicates that the high number of alleles per locus may have occurred since Zélia and IAC-112 are hybrids and Viçosa M-21, UNB-02, SE-013, and Laranjeiras do Sul, are all open-pollinated populations.

The evidence of a third allele at the Umc1653 locus in Viçosa M-21 and SE-013 varieties 
has not been considered or investigated in the present study, but the new alleles that arose from mutations or mitotic recombination events at SSR loci may enlarge the polymorphism level at SSR loci of the popcorn varieties. In the popcorn varieties (69 plants), $2.89 \%$ of plants possess three alleles at the Umc1653 locus. In 101 inbred lines representing tropical, USA, and Canada/ Europe inbred, $0.02 \%$ plants possessed three or more alleles for one microsatellite (Matsuoka et al., 2002). The observation of three or more alleles was justified by authors either as more than one locus that is being amplified in the PCR or alternatively as the result of DNA contamination and/or PCR artifacts, and were treated as missing data. However, the triallelic pattern in SSR loci has also been detected in progenies of sweet corn (Rupp et al., 2009) and has been considered as evidence of chimeras and as a mechanism of genetic diversification in grapevine clones (Hocquigny et al., 2004; Crespan, 2004; Moncada et al., 2006), which have vegetative propagation. Although the reproductive system in maize plants happens by out- or self-crossing, where the chimerism effect has minor importance, the presence of a third allele in SSR locus is indicative of the potential of loci in molecular diversification and variability. Thus, in addition to the Umc1071, Umc2262, Umc2281, Bnlg1083, Umc1077, Umc2226, Umc2343, Umc2115, Umc2116, Umc2047, Umc1664, and Umc1664 loci, where a larger effective number of alleles per locus and higher gene diversity $\left(H_{\mathrm{E}}>0.50\right)$ were observed, the detection of extra alleles may be conferring high genetic diversity also for the Umc1653 locus in the popcorn varieties.

The genetic diversity estimated from the expected mean heterozygosity has not been analyzed in other studies with different popcorn varieties (Wu et al., 2004; Silva et al., 2009) and with inbred lines of popcorn (Dandolini et al., 2008; Trindade et al., 2010). The analysis of SSR loci in popcorn has been principally scored based on the genetic distances, where the estimation of effective number of alleles, the observed heterozygosity and expected heterozygosity were not calculated. The genetic differentiation among popcorn populations has also not been described in other studies. In the present study, the high differentiation among the eight varieties $\left(F_{\mathrm{ST}}=0.3664\right)$ suggests the formation of divergent groups. According to Wright (1978), $F_{\text {ST }}$ values between 0.15 and 0.25 indicate a very high interpopulational divergence level. The genetic relationship between individual plants from different groups may help to select parents for crossing from a base population, and to maximize heterosis. The varieties Zélia and Argentina, e.g., can be considered the most genetically divergent as revealed by 15 SSR loci analyzed in our study. The highest genetic differentiation was evident at Umc2350 locus $\left(F_{\mathrm{ST}}=0.6010\right.$; Table 2$)$; the UMC2350 primer is, therefore, the most suitable primer to characterize the different popcorn varieties based on allele frequencies.

In contrast to the high level of genetic divergence in SSR loci among the eight varieties, an excess of heterozygous was detected in Zélia, IAC-112, UNB-02, and Argentina varieties indicating possibilities of progeny selection in the medium and long-term. High values for proportion of polymorphic loci and observed and expected heterozygosities in most of the varieties (Zelia, IAC-112, UNB-02, and Laranjeiras do Sul) revealed the hybrid (Zélia and IAC-112) and open-pollinated (UNB-02 and Laranjeiras do Sul) conditions for maintenance of these populations. The negative global value of $F_{\text {is }}(-0.0563)$ indicated an excess of $5.63 \%$ of heterozygous plants. The high heterozygosity detected at SSR loci in UNB-02, e.g., may be an important aspect since high heterozygosity would indicate that the plant population probably has a substantial amount of adaptive genetic variation to escape the effects of a control agent that limits the development and maintenance of plants, in contrast to plant populations showing the lowest heterozygosity levels (Argentina and Chile varieties, e.g.). In the our study, the Argentina and 
Chile varieties have shown lower values of observed and expected mean heterozygosity $\left(H_{\mathrm{O}}=\right.$ $0.2889 / H_{\mathrm{E}}=0.2053$ and $H_{\mathrm{O}}=0.2074 / H_{\mathrm{E}}=0.2346$, respectively). The genetic characterization at different popcorn populations from Argentina (Bracco et al., 2009) has shown high and low heterozygosity levels, and a high differentiation between populations from different regions.

For determining varieties, while the selection was apparently strong in some SSR loci (Bnlg1063, Bnlg1083, Umc2350, and Umc2343) showing a lack of heterozygous plants, a significant excess of heterozygous (67.78 and 51.96\%) was detected at Umc1664 and Umc2281 loci, and a moderate excess of heterozygous at Umc1071 (22.4\%), Umc2226 (26.95\%) and Umc $2116(21.15 \%)$ loci. The negative values of $F_{\text {Is }}$ indicate an excess of heterozygous plants. The 15 SSR loci in eight varieties of popcorn have, therefore, relatively low and high levels of genetic variation. Evidence that the domestication and artificial selection for morphological and agronomic characteristics in maize have no significant effect on the level of genetic variation at SSR loci has been indicated by Vigouroux et al. (2005). However, differential levels of genetic variation in SSR loci indicate that particular loci have been the target of artificial selection while in other SSR loci the random allele frequencies are as expected by Hardy-Weinberg equilibrium.

Although SSR loci are usually considered just as evolutionary neutral as DNA markers (Schlotterer and Wiehe, 1999; Schlotterer, 2000), favorable alleles at Bnlg1063, Bnlg1083, Umc2350, and Umc2343 loci, e.g., may be the target of the artificial selection during the formation of populations showing agronomic characteristics favorable for popcorn. The functional significance of a substantial part of SSR loci has been proven by critical tests in various biological phenomena (see review by Li et al., 2002). Subirana and Messeguer (2008) have indicated that microsatellites with different repeated motifs may be structurally related and involved in the determination of chromosome structure.

The functional significance of the apparently strong selection detected in some SSR loci is not known, but our present popcorn collection may be considered as an important reservoir of genes for the generation of different popcorn lines and future production of cultivars. The analysis of genetic structure at eight popcorn varieties using SSR markers was important to identify the SSR loci with high (Umc1071, Umc2262, Umc2281, Bnlg1083, Umc1077, Umc2226, Umc2343, Umc2115, Umc2116, Umc2047, Umc1664, and Umc1664) and low (Umc2350 and Umc1736) levels of heterozygosity, the Umc1653 locus with extra alleles, and varieties showing high heterozygosity (Laranjeiras do Sul, UNB-02, Zélia, and IAC-112), which should be the goal of the selection in the medium- and long-term for the generation of inbred lines and future production of new cultivars. Plants of the Laranjeiras do Sul, UNB-02, Zélia, and IAC-112 varieties may be also recommended for crossing with varieties that present important agronomic characteristics to broaden the genetic basis in popcorn. The selected primers may also be used to check line development, which should be used for future generation of new hybrid cultivars.

The genetic structure analysis of the eight popcorn varieties was especially important to identify the UNB-02 variety as the one that presented the highest effective number of alleles and the highest observed and expected mean heterozygosity values. The UNB-02 variety, as well as Laranjeiras do Sul (a variety of unknown genealogy showing high values for effective number of alleles and mean expected heterozygosity) could be used in medium- or long-term as a source of popcorn inbred lines for future production of new cultivars. Zelia and Argentina varieties also may be used for the generation of inbreed lines since an excess of heterozygous plants 
was detected in both populations. The selected primers (UMC1071, UMC2262, UMC2281, BNLG1083, UMC1077, UMC2226, UMC2343, UMC2115, UMC2116, UMC2047, UMC1664, and UMC1664) in our study may be used to check the genetic stability and the development of the inbred lines, which should be used for future generations of new hybrid cultivars.

\section{ACKNOWLEDGMENTS}

The authors would like to thank CNPq (Conselho Nacional de Pesquisa Ciência e Tecnologia, Brasília, DF), the Araucária Foundation (Curitiba, PR), and CAPES (Coordenação de Aperfeiçoamento de Pessoal de Nível Superior, Brasília, DF, Brazil) for their financial support.

\section{REFERENCES}

Bracco M, Lia VV, Gottlieb AM, Camara HJ, et al. (2009). Genetic diversity in maize landraces from indigenous settlements of Northeastern Argentina. Genetica 135: 39-49.

Crespan M (2004). Evidence on the evolution of polymorphism of microsatellite markers in varieties of Vitis vinifera L. Theor. Appl. Genet. 108: 231-237.

Dandolini TS, Scapim CA, Amaral AT Jr, Mangolin CA, et al. (2008). Genetic divergence in popcorn lines detected by microsatellite markers. Crop. Breed. Appl. Biotechn. 8: 313-320.

Don RH, Cox PT, Wainwright BJ, Baker K, et al. (1991). 'Touchdown' PCR to circumvent spurious priming during gene amplification. Nucleic Acids Res. 19: 4008.

Freitas SP Jr, Amaral AT Jr, Pereira MG, Cruz CD, et al. (2006). Capacidade combinatória em milho pipoca por meio de dialelo circulante. Pesq. Agropec. Brasil. 41: 1599-1607.

Hocquigny S, Pelsy F, Dumas V, Kindt S, et al. (2004). Diversification within grapevine cultivars goes through chimeric states. Genome 47: 579-589.

Hoisington DM, Khairallah D and Gonzalez-de-Leon L (1994). Laboratory Protocols: CIMMYT Applied Molecular Genetics Laboratory. 2nd edn. DF: CIMMYT, Mexico.

Leal AA, Mangolin CA, do Amaral ATJ, Goncalves LS, et al. (2010). Efficiency of RAPD versus SSR markers for determining genetic diversity among popcorn lines. Genet. Mol. Res. 9: 9-18.

Leonello LAF, Cazetta DA and Fornasieri Filho D (2009). Características agronômicas e qualidade comercial de cultivares de milho pipoca em alta população. Acta Sci. Agron. 31: 215-220.

Li YC, Korol AB, Fahima T, Beiles A, et al. (2002). Microsatellites: genomic distribution, putative functions and mutational mechanisms: a review. Mol. Ecol. 11: 2453-2465.

Li YL, Lv DB, Wang YZ, Chen SJ, et al. (2004). Study on the genetic diversity of popcorn inbreeds and their germoplasm relationship with normal corn inbreeds using SSR markers. Maydica 49: 327-333.

Liu K, Goodman M, Muse S, Smith JS, et al. (2003). Genetic structure and diversity among maize inbred lines as inferred from DNA microsatellites. Genetics 165: 2117-2128.

Liu YJ, Huang YB, Rong TZ, Tian ML, et al. (2005). Comparative analysis of genetic diversity in land-races of waxy maize from Yunnan and Guizhou using SSR markers. Sci. Agric. Sin. 4: 648-653.

Matsuoka Y, Vigouroux Y, Goodman MM, Sanchez GJ, et al. (2002). A single domestication for maize shown by multilocus microsatellite genotyping. Proc. Nat. Acad. Sci. U. S. A. 99: 6080-6084.

Miranda GV, Souza LV, Galvão JCC, Guimarães LJM, et al. (2008). Genetic variability and heterotic groups of Brazilian popcorn populations. Euphytica 162: 431-440.

Moncada X, Pelsy F, Merdinoglu D and Hinrichsen P (2006). Genetic diversity and geographical dispersal in grapevine clones revealed by microsatellite markers. Genome 49: 1459-1472.

Nei M (1973). Analysis of gene diversity in subdivided populations. Proc. Natl. Acad. Sci. U. S. A. 70: 3321-3323.

Pereira LK, Scapim JB, Mangolin CA, Machado MFPS, et al. (2008). Heterozygosity following half-sib recurrent selection in popcorn using isoenzyme markers. Elect. J. Biotechn. 11: 107-115.

Qi-Lun Y, Ping F, Ke-Cheng K and Guang-Tang P (2008). Genetic diversity based on SSR markers in maize (Zea mays L.) landraces from Wuling mountain region in China. J. Genet. 87: 287-291.

Rangel RM, Amaral AT Jr, Scapim CA, Freitas SP Jr, et al. (2008). Genetic parameters in parents and hybrids of circulant diallel in popcorn. Genet. Mol. Res. 7: 1020-1030.

Rupp JV, Mangolin CA, Scapim CA and Machado MFPS (2009). Genetic structure and diversity among sweet corn (Su1- 
germplasm) progenies using SSR markers. Maydica 54: 125-132.

Sambrook J, Fritsch EF and Maniatis T (1989). Molecular Cloning: A Laboratory Manual. 2nd edn. Cold Spring Harbor Laboratory, Cold Spring Harbor Laboratory Press, New York.

Santos FS, Amaral AT Jr, Freitas SP Jr, Rangel RM, et al. (2008). Genetic gain prediction of the third recurrent selection cycle in a popcorn population. Acta Sci. Agron. 30: 651-655.

Scapim CA, Braccini AL, Pinto RJB, Amaral AT Jr, et al. (2006). Componentes genéticos de médias e depressão por endogamia em populações de milho-pipoca. Cienc. Rural 36: 36-41.

Schlotterer C (2000). Evolutionary dynamics of microsatellite DNA. Chromosoma 109: 365-371.

Schlotterer C and Wiehe T (1999). Microsatellites, A Neutral Marker to Infer Selective Sweeps. In: Microsatellites: Evolution and Applications (Goldstein DB and Schlötterer C, eds.). Oxford University Press, Oxford, 238-247.

Silva TA, Pinto RJB, Scapim CA, Mangolin CA, et al. (2009). Genetic divergence in popcorn genotypes using microsatellites in bulk genomic DNA. Crop. Breed. Appl. Biotechn. 9: 31-36.

Subirana JA and Messeguer X (2008). Structural families of genomic microsatellites. Gene 408: 124-132.

Trindade APR, Pinto RJB, Amaral AT Jr, Mangolin CA, et al. (2010). Genetic diversity of breeding popcorn lines determined by SSR markers. Elect. J. Biotechn. 13: 1-9.

Vieira RA, Souza Neto IL, Bignotto LS, Cruz CD, et al. (2009). Heterotic parametrization for economically important traits in popcorn. Acta Sci. Agron. 31: 411-419.

Vigouroux Y, Mitchell S, Matsuoka Y, Hamblin M, et al. (2005). An analysis of genetic diversity across the maize genome using microsatellites. Genetics 169: 1617-1630.

Vilela FO, Amaral AT Jr, Pereira MG, Scapim CA, et al. (2008). Effect of recurrent selection on the genetic variability of the UNB-2U popcorn population using RAPD markers. Acta Sci. Agron. 30: 25-30.

Wright S (1965). The interpretation of population structure by F-statistics with special regard to systems of mating. Evolution 19: 395-420.

Wright S (1978). Variability Within and Among Populations. In: Evolution and the Genetics of Populations (Wright S, ed.). University of Chicago Press, Chicago, 580.

Wu YS, Zheng YL, Sun R, Wu SY, et al. (2004). Genetic diversity of waxy corn and popcorn landraces in Yunnan by SSR markers. Acta Agron. Sin. 30: 36-42.

Yeh FC, Yang R and Boyle T (1999). Microsoft Window-based freeware for population genetic analysis. Popgene Version 1.31. University of Alberta, Centro for International Forestry Research, Edmonton. 